\section{P1.033 THE VAGINAL MICROBIOME AND ITS CLINICAL CORRELATES IN A COHORT OF AFRICAN SEX WORKERS}

doi:10.1136/sextrans-2013-051184.0254

'H Borgdorff, ${ }^{2} \mathrm{E}$ Tsivtsivadze, ${ }^{3} \mathrm{R}$ Verhelst, ${ }^{2} \mathrm{~F}$ H Schuren, ${ }^{4} \mathrm{M}$ Marzorati, $5 \mathrm{~J} \mathrm{H} \mathrm{H} \mathrm{M} \mathrm{van}$ de Wijgert. 'Amsterdam Institute for Global Health and Development (AIGHD) and Academic Medical Center, Department of Global Health, Amsterdam, The Netherlands; ${ }^{2} T N O$ Microbiology \& Systems Biology, Zeist, The Netherlands; ${ }^{3}$ International Center for Reproductive Heath (ICRH), Ghent University, Ghent, Belgium; "Faculty of Bioscience Engineering, Laboratory of Microbial Ecology and Technology (LabMET), Ghent University, Ghent, Belgium; ${ }^{5}$ University of Liverpool, Institute of Infection and Global Health, Liverpool, UK

Background Although Sub-Saharan Africa is one of the most important areas in the world to study the complex relationships between the vaginal microbiome and reproductive health outcomes, data are limited.

Methods Endocervical samples of 174 female sex workers in Kigali, Rwanda, were analysed cross-sectionally using a phylogenetic microarray specifically designed for the cervicovaginal microbiome. Women with sexually transmitted infections (STI) were purposefully oversampled. Two hundred fifty one probes were used for co-regularised spectral clustering analysis and 123 probes (specific at species or genus level) to describe the vaginal microbiome clusters. Demographic, behavioural, and clinical correlates of the clusters were also determined.

Results The prevalence of HIV (36\%) and other STIs (bacterial STI $46 \%$, HPV $48 \%$, and HSV-2 $78 \%$ ) in the analysis sample were high by design. Six distinct vaginal microbiome clusters were identified. Two clusters were dominated by Lactobacillus crispatus and $L$. iners, respectively, and were associated with a Nugent score of 0-3. Three clusters were dominated by Gardnerella vaginalis, Atopobium spp. and Prevotella spp in different compositions, and were associated with a Nugent score of 7-10. The sixth cluster, also dominated by anaerobic bacteria, was not associated with a particular Nugent score category. Women belonging to the L. crispatus cluster were significantly less likely to have bacterial (0\% compared to $32-67 \%)$ and viral STIs (36\% compared to $89-100 \%)$ than women in the other 5 clusters.

Conclusion In this sample of African sex workers with a high prevalence of HIV and STIs, six vaginal microbiome clusters were identified. Sex workers with a vaginal microbiome dominated by L. crispatus (but not L. iners) did not have bacterial STIs and were less likely to have viral STIs than women with other microbiome compositions. Longitudinal studies are needed to determine the temporal relationships between the vaginal microbiome and various STIs.

\section{P1.034 BIOMARKERS OF CIGARETTE SMOKING AND ASSOCIATION WITH THE VAGINAL MICROBIOTA}

doi:10.1136/sextrans-2013-051184.0255

'R M Brotman, 'J Ravel, 'P Gajer, 'D Fadrosh, 'E F Mongodin, 'E D Glover, ${ }^{3} \mathrm{~J}$ M Rath. 'University of Maryland School of Medicine, Institute for Genome Sciences, Baltimore, MD, United States; 'University of Maryland School of Public Health, Department of Behavioral and Community Health, College Park, MD, United States; ${ }^{3}$ Legacy Foundation, District of Columbia, WA, United States

Background Smoking has been identified in observational studies as a risk factor for bacterial vaginosis (BV). The anti-estrogenic effect of smoking and trace amounts of benzo[a]pyrene diol epoxide (BPDE) may predispose women to BV. BPDE increases bacteriophage induction in Lactobacillus spp. and is found in the vaginal secretions of smokers.

Methods Vaginal microbiota of smokers versus non-smokers were compared in a cross-sectional study (Phase A, $n=40$ ). In Phase B $(\mathrm{n}=9)$ participants underwent behavioural counselling for 12-weeks and were offered nicotine patches to encourage cessation. In both Phases, participants self-collected mid-vaginal swabs (daily, Phase B) and completed behavioural diaries. Vaginal bacterial composition was characterised by pyrosequencing of barcoded 16S rRNA genes (27F-mixture and 534R, V1-V3). Vaginal smears were Nugent Gram stained. Smoking status was evaluated (weekly, Phase B) using the semi-quantitative $\mathrm{NicAlert}^{\circledR}$ saliva cotinine test and carbon monoxide (CO) exhalation.

Results Vaginal microbiota clustered into 3 community state types (CSTs); 2 dominated by Lactobacillus (L. iners, L. crispatus), and 1 lacking significant numbers of Lactobacillus spp. and characterised by anaerobes (termed CST-IV). There were statistically significant trends for increasing cotinine concentration and $\mathrm{CO}$ with increasing Nugent score. Smokers were 7-fold more likely to be assigned CST-IV than the L. crispatus-dominated CST (OR:7.22, 95\% CI: 1.44-36.22). 4 women completed Phase B. 1 of 3 women who entered the smoking cessation with high Nugent scores demonstrated a switch from CST-IV-dominated communities to a L.inersdominated profile with a concomitant drop in Nugent scores, coinciding with lower dose nicotine patches. The other 2 women fluctuated between CST-IV and L. iners-dominated CSTs. The fourth woman had low Nugent scores with L. crispatus-dominated CSTs throughout.

Conclusion Smokers have a lower proportion of Lactobacillus spp. Smoking cessation may shift some women to a Lactobacillus-dominated state and decrease the risk of BV. Larger studies are needed to confirm these findings.

\section{P1.035 EXPRESSION, PURIFICATION AND CHARACTERIZATION OF ANTI-V3 HUMAN SCFVS AGAINST HIV-1 CLADE C}

doi:10.1136/sextrans-2013-051184.0256

'R Kumar, 'R Andrabi, 'A Tiwari, ' $\mathrm{N}$ Wig, ${ }^{2} \mathrm{~S}$ Sinha, ${ }^{1} \mathrm{~K}$ Luthra. ${ }^{1}$ All India Institute of medical sciences, Delhi, India; ${ }^{2}$ National Brain Research Centre, Manesar, Harayana, India

Background Production of human monoclonal antibodies that shows broadly neutralising activity is needed for the prevention of HIV-1. Here we devised a novel approach and produced 11 different human scFvs against the V3 region of HIV-1 envelope. Method: The peripheral blood mononuclear cells (PBMCs) were isolated from an HIV-1 clade C infected drug naïve Indian patient whose plasma exhibited neutralising antibodies against a panel of viruses. PBMCs were EBV transformed in 96 well plate and then expanded into 24 well stage followed by 6 well and finally to flask stage. The transformed cells (wells) were screened against HIV-1 consensus C V3 peptide at each stage and only the positive cells (wells) were carried onto the next stage. Total RNA from these enriched positive antibody secreting cells at the flask stage was isolated and cDNA was synthesised. Results: By EBV transformation and preselection of V3 specific clones we successfully constructed a small phage library. One round of bio panning was done against HIV- 1 consensus C and B V3 peptides. Randomly, 40 clones were selected and checked for their binding. Out of 40 clones, 13 showed positivity in phage ELISA. DNA fingerprinting analysis using BstN1 followed by sequencing showed that 11 out of 13 clones were distinct. Expression of positive clones was tested by SDS-PAGE and Western blot. All the 11 anti -V3 scFvs showed cross-reactivity against both the V3 peptides and did not show any reactivity against other unrelated peptides. The scFvs showed varying degrees of neutralisation against tier 1 and tier 2 viruses and cross neutralising activity against clade $\mathrm{A}, \mathrm{B}$ and $\mathrm{C}$ viruses.

Conclusion This is the first study to generate anti-V3 scFvs against HIV-1 Clade C. Further assessment of the neutralisation efficiency of these scFvs would reveal their potential for passive immunotherapy. 